\title{
Screening corrections to nuclear reaction rates in low-mass stars and brown dwarfs
}

\author{
August Wierling* \\ Universität Rostock, Institut für Physik, 18051 Rostock, Germany \\ E-mail: august.wierling@uni-rostock.de
}

\begin{abstract}
Plasma corrections to reaction rates for deuterium burning in brown dwarfs and low-mass stars are revisited. A quantum-statistical approach is outlined. With the help of thermo-dynamic Green's functions, many-body and quantum effects are systematically taken into account. Specifically, the relevance of dynamical screening as well as degeneracy effects are discussed.
\end{abstract}

11th Symposium on Nuclei in the Cosmos, NIC XI

July 19-23, 2010

Heidelberg, Germany

${ }^{*}$ Speaker. 


\section{Deuterium burning in low-mass stars}

Screening corrections to nuclear reaction rates play an important rôle in stellar modeling and stellar evolution [1]. For hot and dilute plasmas, these corrections have already been studied in the pioneering work of Salpeter [2] and are well understood. However, for relatively cold and dense plasmas, more sophisticated theories accounting for many-body effects as well as quantum statistics are indispensable [3].

The existence of brown dwarfs has been conjectured in the earlier 1960s [4]. Since the first definitive identification of the genuine brown dwarf Gl 229 B by Nakajima [5], the study of brown dwarfs has progressed enormously due to observational efforts in infrared spectroscopy. Theoretical modeling suggests, that these stars have much cooler and denser core plasma conditions compared to e.g. our sun [6].

Brown dwarfs and very low mass stars generate their energy via a truncated pp-chain given by the reactions , cf. Ref. [6],

$$
\begin{aligned}
p+d & \rightarrow{ }^{3} \mathrm{He}+\gamma, \\
p+p & \rightarrow d+e^{+}+v_{e}, \\
p+e^{-}+p & \rightarrow d+v_{e} .
\end{aligned}
$$

The first reaction converts primordial deuterium with a $Q$-value of $Q=5.494 \mathrm{MeV}$. Recent values for the isolated nuclear reaction rates can be found in Refs. [7]. However, these reaction rates are modified in the stellar environment due to many-body effects such as screening and degeneracy. The importance of these corrections can be estimated from the non-ideality parameter $\Gamma_{c}=Z_{c}^{2} e^{2} /\left(4 \pi \varepsilon_{0} k_{B} T\right)\left(4 \pi n_{c} / 3\right)^{1 / 3}$ and the degeneracy parameter $\theta_{c}=2 m_{c} k_{B} T / \hbar^{2}\left(3 \pi^{2} n_{c}\right)^{-2 / 3}$ for a multi-component plasma with particle-densities $n_{c}$ and temperature $T, c$ being the index labeling the species. As a specific example, we consider the core conditions given by Burrows et al. [8], model $\mathrm{X}$. This model assumes solar metallicity, a helium fraction of $Y_{\alpha}=0.25$, and a deuterium fraction of $Y_{d}=2 \times 10^{-5}$. In Fig. 1, we illustrate the parameters $\Gamma_{e}$ and $\Theta_{e}$ for these conditions. Note, that for very low-mass stars the parameters $\Gamma_{e}$ and $\Gamma_{p}$ exceed unity and $\Gamma_{\alpha}$ approaches unity, see also Tab. 1 . Also, the degeneracy parameter $\theta_{e}$ of electrons is smaller than unity at low masses. Thus, we expect that interaction as well as degeneracy effects must be accounted for. In the following, we only use the data for an age of 0.003 Gyrs since deuterium burning is occuring only in the early phase of the brown dwarf evolution.

\section{Many-body approach to screening corrections}

The standard approach to estimate screening corrections was developed in Ref. [9] and extended by Mitler [10]. It is based on classical statistics and evaluates the so-called screening potential, see also Ref. [3]. It accounts for many-body effects, but ignores quantum effects, dynamical effects, and (partial) degeneracy. Here, we outline a more systematic approach based on quantum statistics using thermodynamic Green's functions. As was shown in Ref. [11], the reaction rate can be obtained from a Green's function approach, cf. Ref. [12]. In particular, for binary reactions $1+2 \rightarrow 3+4$ and neglecting correlations between the in-coming channel and the out-going 


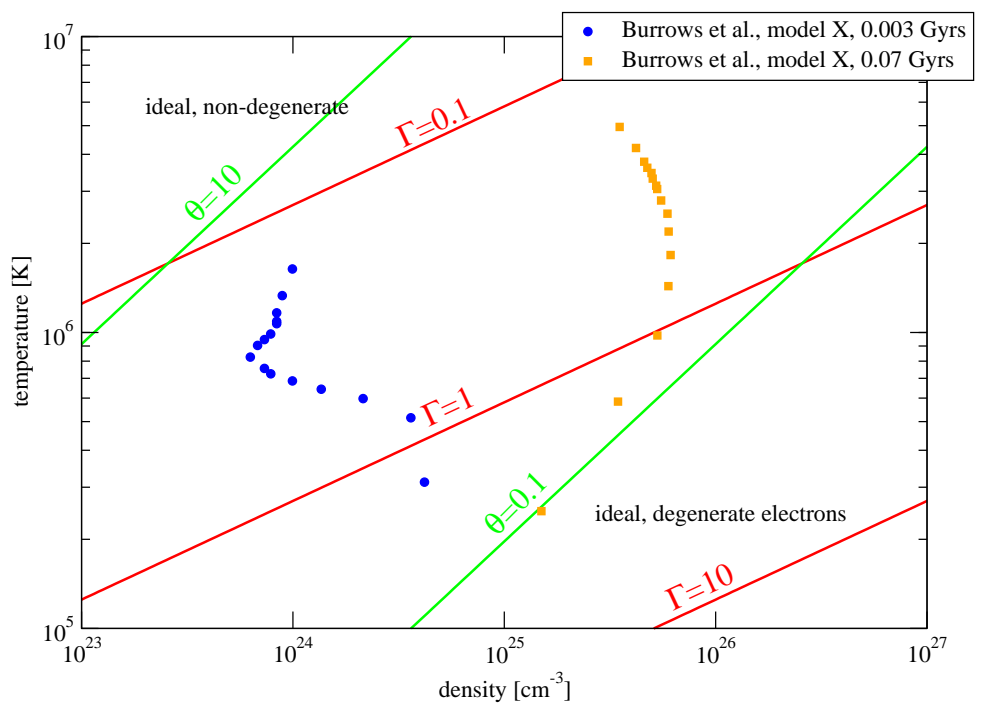

Figure 1: Density and temperature conditions for the electron gas at core conditions in the model $\mathrm{X}$ of Ref. [8]. Samples for a an age of 0.003 Gyrs and 0.07 Gyrs are shown. Lines for constant degeneracy parameter $\theta_{e}$ and non-ideality parameter $\Gamma_{e}$ are also indicated.

channel, we have for the rate $R$

$$
\begin{aligned}
R_{1+2 \rightarrow 3+4}(Q)= & \sum_{p_{\text {in }}, p_{\text {out }}} \int_{-\infty}^{\infty} \frac{d \hbar \omega}{2 \pi}\left|V_{\text {react }}\left(p_{\text {in }}, p_{\text {out }}\right)\right|^{2} \\
& \times n_{B}\left(\hbar \omega-\mu_{1}-\mu_{2}\right)\left(1+n_{B}\left(\hbar \omega+Q-\mu_{3}-\mu_{4}\right)\right) A_{12}\left(p_{\text {in }}, \hbar \omega\right) A_{34}\left(p_{\text {out }}, \hbar \omega+Q\right)
\end{aligned}
$$

According to this formula, the rates splits into a) the nuclear information $\left|V_{\text {react }}\left(p_{\text {in }}, p_{\text {out }}\right)\right|^{2}$, i.e. the cross section at a given energy, b) statistical information via the two-particle Bose distribution $n_{B}(\omega)$ and the chemical potentials $\mu_{1}, \mu_{2}$ it contains, and c) correlations for the propagation of two-particle states represented by the two-particle spectral functions $A_{12}$ and $A_{34}$. The summation is performed with respect to the incoming and outgoing momenta $p_{\text {in }}, p_{\text {out }}$ of the particles involved. The two-particle spectral functions can be determined by solving an in-medium two-particle equation being a generalization of the Lippmann-Schwinger equation, e.g. for the incoming channel,

$$
\begin{aligned}
& \left(E_{p}(1)+E_{d}(2)+\Delta_{p, d}^{\mathrm{eff}}(12, z)-z\right) G_{p, d}\left(121^{\prime} 2^{\prime}, z\right) \\
& +\sum_{\overline{1} \overline{2}} V_{p, d}^{\mathrm{eff}}(12 \overline{1} \overline{2}, z) G_{p, d}\left(\overline{1} \overline{2} 1^{\prime} 2^{\prime}, z\right)=i \delta_{11^{\prime}} \delta_{22^{\prime}} N_{p, d}(12)
\end{aligned}
$$

with $121^{\prime} 2^{\prime} \overline{1} \overline{2}$ being momenta, $E_{p}(1), E_{d}(2)$ indicating kinetic energies. $\Delta_{p, d}^{\text {eff }}(12, z)$ and $V_{p, d}^{\text {eff }}(12 \overline{1} \overline{2}, z)$ are effective self energy and effective interaction due to the surrounding particles in the plasma. The Green's function $G_{p, d}$ and the spectral function $A_{p, d}$ are connected via $A_{p, d}(\omega)=G_{p, d}(\omega+$ $i \varepsilon)-G_{p, d}(\omega-i \varepsilon)$. Single-particle corrections are determined by solving the Dyson equation, see for details Ref. [12]. Screening as the most important many-body effect is included by defining a dynamical screened interaction $V_{s}(q, \omega)=V(q) / \varepsilon(q, \omega)$ where $\varepsilon(q, \omega)$ is the dielectric function. Here, $V(q)$ is the Coulomb potential in momentum space representation and $\omega$ is a frequency. For $\omega=0, V_{s}(q, \omega=0)$ reduces to the static screened potential. The dielectric function is treated in 
Random Phase approximation (RPA) taking full account of quantum and degeneracy effects, cf. Ref. $[13,14]$. Note that $V_{S}$ is a dynamical quantity taking care of the retardation effects due to the motion of particles.

\section{Assessing screening corrections in low-mass stars}

The approach outlined above is quite involved and results will be presented in detail in a forthcoming paper, see also Ref. [11]. Here, we will give some simple estimates for the importance of many-body effects. These shall serve only as a guide to pin down plasma conditions to be considered with the more elaborated approach. To this end, we consider Salpeter screening, i.e. we assume, that the screening correction $f\left(n_{c}, T\right)$ is given by the total screening length $\kappa$ as $f\left(n_{c}, T\right)=$ $\exp \left(e^{2} \kappa / 4 \pi \varepsilon_{0}\right)$. We define a generalized screening length for the electrons by

$$
k_{s, e}^{2}=\frac{1}{2} k_{T F}^{2} \theta_{e}^{1 / 2} F_{-1 / 2}\left(\mu_{e} / k_{B} T_{e}\right),
$$

where $k_{T F}=\left(3 \theta_{e} / 2\right)^{1 / 2} \kappa_{D, e}$ is the Thomas-Fermi length, $F_{-1 / 2}$ the Fermi-Dirac function with index $-1 / 2$ and $\mu_{e}$ the chemical potential for electrons at density $n_{e}$ and temperature $T_{e}$. Note that this expression reduces to the traditional Debye-screening length $\kappa_{D, e}=\left(e^{2} n_{e} / \varepsilon_{0} k_{B} T\right)^{1 / 2}$ for a non-degenerate plasma, while $k_{s, e}$ coincides with $k_{T F}$ for highly degenerate plasmas. The total screening length $\kappa$ is obtained as

$$
\kappa^{2}=k_{s, e}^{2}+x_{p} \kappa_{D, p}^{2}+x_{d} \kappa_{D, d}^{2},
$$

where $\kappa_{D, p}$ and $\kappa_{D, d}$ are the Debye screening length for protons and deuterons, respectively. $x_{p}$ and $x_{d}$ are correction factors accounting for a partial screening by protons and deuterons due to low mobility of ions as compared to electrons. Here, we take the numerical value of $x_{p}=x_{d}=0.865$ from Ref. [16] where is has been obtained within the same theoretical approach outlined above.

We present our results in Tab. 1 . The estimate $\bar{f}$ for the screening corrections normalized to Debye screening is shown as a function of the mass of the star. Accounting for degeneracy leads to a decrease in the electron screening length $k_{s, e}$ at low masses as compared to Debye screening given by $\kappa_{D, e}$. Already for $M / M_{\text {sun }} \leq 0.04$, deviations are larger than $10 \%$. A reduction of the screening length corresponds to a more Coulomb-like potential, which is harder to penetrate by the reacting particles. Thus, the rate is reduced compared to a description ignoring deneraracy effects. Dynamic screening effects given by $x_{p}$ and $x_{d}$ act in the same vein. However, significant deviations in $\bar{f}$ arise only for very small masses. This is due to the fact, that the core temperature is still large, diminishing effects in the screening length.

\section{Acknowledgments}

This work was supported by the Deutsche Forschungsgemeinschaft via project RO905/30-1.

\section{References}

[1] D.D. Clayton, Principles of Stellar Evolution and Nuclear Synthesis, McGraw-Hill, New York 1968; C.E. Rolfs and W.S. Rodney, Cauldrons in the Cosmos, University of Chicago Press, Chicago 1988. 


\begin{tabular}{cccccccc}
$M / M_{\text {sun }}$ & $\theta_{e}$ & $\Gamma_{p}$ & $\Gamma_{\alpha}$ & $\kappa_{D, e} a_{B}$ & $k_{s, e} a_{B}$ & $\kappa_{D, p} a_{B}$ & $\bar{f}\left(n_{c}, T\right)$ \\
\hline 0.2 & 3.89 & 0.17 & 0.27 & 0.60 & 0.59 & 0.55 & 0.996 \\
0.15 & 3.40 & 0.21 & 0.32 & 0.63 & 0.61 & 0.58 & 0.995 \\
0.1 & 2.74 & 0.24 & 0.42 & 0.68 & 0.66 & 0.63 & 0.992 \\
0.09 & 2.76 & 0.25 & 0.44 & 0.67 & 0.65 & 0.62 & 0.992 \\
0.08 & 2.66 & 0.27 & 0.46 & 0.67 & 0.65 & 0.62 & 0.991 \\
0.07 & 2.23 & 0.30 & 0.53 & 0.75 & 0.72 & 0.69 & 0.988 \\
0.06 & 2.01 & 0.33 & 0.57 & 0.80 & 0.76 & 0.74 & 0.985 \\
0.05 & 1.63 & 0.37 & 0.65 & 0.92 & 0.87 & 0.85 & 0.980 \\
0.04 & 1.24 & 0.44 & 0.77 & 1.12 & 1.02 & 1.03 & 0.970 \\
0.03 & 0.85 & 0.55 & 0.97 & 1.45 & 1.26 & 1.34 & 0.946 \\
0.02 & 0.52 & 0.76 & 1.33 & 2.03 & 1.57 & 1.88 & 0.880 \\
0.01 & 0.28 & 1.32 & 2.31 & 2.81 & 1.76 & 2.60 & 0.663 \\
\hline \hline
\end{tabular}

Table 1: Estimate of the screening correction $\bar{f}\left(n_{c}, T\right)$ normalized to Debye screening as a function of the mass of the low mass star in units of solar masses. Density and temperature conditions are taken from model $\mathrm{X}$ in Ref. [8]. Shown also: the non-ideality parameter for protons and $\alpha$-particles $\Gamma_{p}, \Gamma_{\alpha}$, the degeneracy parameter for electrons $\theta_{e}$, the generalized electron screening length $k_{s, e}$, the Debye-screening length for electrons $\kappa_{D, e}$ and protons $\kappa_{D, p}$. For all stars, an age of 0.003 Gyrs and a $\alpha$-particle fraction $Y_{\alpha}=0.25$ is assumed. $a_{B}$ is the Bohr radius.

[2] E.E. Salpeter, Australian J. Phys. 7 (1954) 373.

[3] S. Ichimaru, Rev. Mod. Phys. 68 (1993) 255.

[4] S.S. Kumar, Astrophys. J. 137 (1963) 1121; C. Hiyashi and T. Nakano, Prog. Theor. Phys. 30 (1963) 460.

[5] T. Nakajima, Nature 378 (1995) 464.

[6] A. Burrows, W.B. Hubbard, J.I. Lunine, and J. Liebert, Rev. Mod. Phys. 73 (2001) 719.

[7] G.R. Caughlan and W.A. Fowler, Atom. Data Nucl. Data Tables 40 (1988) 283; P. Descouvement, A. Adahchour, C. Angulo, A. Coc, and E. Vangioni-Flam, Atom. Data Nucl. Data Tables 88 (2004) 203.

[8] A. Burrows, W.B. Hubbard, D. Saumon, and J.I. Lunine, Astrophys. J. 406 (1993) 158.

[9] H.C. Graboske, H.E. DeWitt, A.S. Grossman, and M.S. Cooper, Astrophys. J. 181 (1973) 457.

[10] H.E. Mitler, Astrophys. J. 212 (1977) 513.

[11] A. Wierling, T. Millat, G. Röpke, Nucl. Phys. A 688 (2001) 569.

[12] W.D. Kraeft, D. Kremp, W. Ebeling, and G. Röpke, Quantum Statistics of Charged Particle Systems, Akademie-Verlag, Berlin 1986; G.D. Mahan, Many-Particle Physics, Plenum, New York 1990.

[13] N.R. Arista and W. Brandt, Phys. Rev. A 29 (1984) 1471.

[14] A. Wierling, Phys. Plasmas 16 (2009) 112105.

[15] A. Wierling and G. Röpke, Contrib. Plasma Phys. 38 (1998) 513.

[16] G. Röpke, Phys. Rev. A 38 (1988) 3001. 